\title{
Percepção monocular da profundidade ou relevo na ilusão da máscara côncava na esquizofrenia
}

\author{
Arthur Alves \\ Escola Municipal "Raio de Sol" \\ Maria Amélia Cesari Quaglia \\ Universidade Federal de São João del Rei \\ Lívia da Silva Bachett \\ Universidade de São Paulo \\ Marcos Santos de Oliveira \\ Universidade Federal de São João del Rei
}

\begin{abstract}
Resumo
Este trabalho foi desenvolvido com o propósito de investigar a percepção monocular da profundidade ou relevo da máscara côncava por 29 indivíduos saudáveis, sete indivíduos com esquizofrenia sob uso de antipsicótico por um período inferior ou igual a quatro semanas e 29 sob uso de antipsicótico por um período superior a quatro semanas. Os três grupos classificaram o reverso de uma máscara policromada em duas situações de iluminação, por cima e por baixo. Os resultados indicaram que a maioria dos indivíduos com esquizofrenia inverteu a profundidade da máscara côncava na condição de observação monocular e perceberam-na como convexa, sendo, portanto, suscetíveis à ilusão da máscara côncava. Os indivíduos com esquizofrenia sob uso de medicação antipsicótica pelo período superior a quatro semanas estimaram a convexidade da máscara côncava iluminada por cima em menor comprimento comparados aos indivíduos saudáveis.
\end{abstract}

Palavras-chave: máscara côncava; esquizofrenia; percepção visual.

\begin{abstract}
Monocular depth perception or relief in the hollow-face illusion in the schizophrenia. The goal of the present study was to investigate the monocular perception of the depth or relief of the concave mask by 29 healthy subjects: seven individuals with schizophrenia under use of antipsychotic for a period less than or equal to four week and 29 under use of antipsychotic for a period bigger than four week. The three groups classified the reverse of a mask in two situations polychromatic illumination from above and below. The results showed most of the subjects with schizophrenia made the monocular depth inversion of a concave mask and perceived it as convex. The individuals with schizophrenia on antipsychotic medication for longer than four weeks period estimated the convexity of illuminated concave mask over into shorter length compared to healthy individuals and therefore susceptible to the illusion of concave mask.
\end{abstract}

Keywords: hollow-mask; schizophrenia; visual inversion.

\section{Resumen}

Percepción monocular de la profundidad o relieve en la ilusión de la mascara hueca en la esquizofrenia. Este estudio fue desarrollado con el objetivo de investigar la percepción monocular de la profundidad o relieve de una máscara hueca por 29 sujetos saludables, 7 con esquizofrenia bajo uso de medicación antipsicótica durante un período igual o inferior a 4 semanas y 29 bajo uso de medicación antipsicótica por un período mayor de 4 semanas. Los 3 grupos clasificaron el reverso de una máscara hueca policromada en dos situaciones de iluminación, por encima y por debajo de la cabeza. Los resultados mostraron que la mayoría de los sujetos con esquizofrenia han invertido el fondo cóncavo de la máscara hueca en la condición de observación monocular y percibieron-no como convexo. Ellos fueron susceptibles a la ilusión de la máscara hueca. Además, los sujetos con esquizofrenia en uso de medicación antipsicótica durante un periodo mayor de 4 semanas juzgaron la convexidad de la máscara hueca iluminada por encima en menor dimensión, en comparación a los saludables.

Palabras clave: máscara hueca; esquizofrenia; percepción visual. 
A esquizofrenia é uma condição psiquiátrica que interfere nos pensamentos, emoções, motivações, comportamento motor e cognição (Walther et al., 2009). A esquizofrenia também interfere na percepção visual, mais especificamente incapacitando o indivíduo de realizar a inversão visual da profundidade (Dima et al., 2009). Quando objetos côncavos são observados por indivíduos saudáveis ocorre uma inversão visual da profundidade e o que é côncavo é percebido como convexo, provavelmente porque as convexidades são mais frequentes na natureza (Kleffner \& Ramachandran, 1992). A ilusão da máscara côncava é um exemplo de objeto no qual ocorre uma inversão visual da profundidade. Em indivíduos saudáveis, a ilusão acontece quando um molde côncavo ou o reverso de uma máscara da face, observado a certa distância, provoca uma inversão da percepção da profundidade e o reverso passa a ser visto como uma face em relevo, convexa. Esse fenômeno da inversão da percepção da profundidade é robusto mesmo sob variadas condições de apresentação da máscara côncava (Quaglia \& Fukusima, 2009). Algumas condições de apresentação da máscara côncava facilitam a inversão visual da profundidade como: a coloração com tonalidades próximas às da face humana (Hill \& Bruce, 1993), a apresentação da máscara côncava na posição vertical, a direção da fonte de iluminação percebida por cima da máscara côncava (Hill \& Bruce, 1993; Yoshida, 2006) e a máscara côncava apresentada na condição de observação monocular em contraposição a apresentada na condição binocular (Hill \& Bruce, 1993).

Essa inversão da profundidade da máscara côncava é compreendida como um processo que envolve a geração de hipóteses sobre a forma tridimensional de faces, mediado pelos conhecimentos conceitual e perceptivo, presentes no processamento de cima para baixo, top-down, que se sobrepõe aos estímulos sensoriais que chegam ao cérebro, processo bottom -up, de baixo para cima (Gregory, 1997). Ordinariamente, no processamento bottom-up ocorre uma integração das informações, estímulos, características, ou atributos básicos dos objetos que chegam ao sistema visual. Estes atributos são, então, organizados por mecanismos de análise e síntese cerebrais que resultam na percepção de formas e reconhecimento dos objetos, processamento top-down (Cruz, 2000). Mas na ilusão da máscara côncava, segundo Gregory (1997), a preponderância dos processos de alta ordem é de tal intensidade que contraria as pistas visuais de profundidade e as informações estereoscópicas, apreendidas pelos sentidos, que indicam a concavidade do objeto, bottom-up. No entanto, indivíduos com esquizofrenia tendem a não perceber a ilusão da máscara côncava. O reverso de uma máscara ou molde de uma face humana é corretamente percebido por esses indivíduos como sendo côncavo, não ocorrendo automaticamente a inversão de sua profundidade (Dima et al., 2009).

Alguns estudos investigaram esse fenômeno da ilusão da máscara côncava em indivíduos com esquizofrenia. No estudo de Schneider et al. (1996) os indivíduos com esquizofrenia sofreram prejuízo na realização da inversão binocular da profundidade de estereogramas de objetos com alto e baixo grau de familiaridade. Resultados semelhantes foram obtidos nos estudos de Schneideret al. (2002) e Koethe et al. (2009) nos quais investigaram a inversão binocular da profundidade empregando imagens estereoscópicas de faces humanas. No estudo de Schneider et al. (2002), o uso de medicação antipsicótica após a terceira semana restabeleceu essa capacidade. Também nos estudos de Dima et al. (2009), Dima, Dietrich, Dillo e Emrich (2010) e Dima, Dillo, Bonnemann, Emrich e Dietrich (2011), os indivíduos com esquizofrenia tiveram dificuldades para realizar a inversão binocular da profundidade de imagens estereoscópicas de faces.

Objetivando ampliar o conhecimento acerca da percepção visual da profundidade ou relevo da máscara côncava em indivíduos com esquizofrenia, o presente estudo teve como objetivo geral verificar a percepção da profundidade ou relevo da máscara côncava em indivíduos com esquizofrenia. E teve como objetivos específicos verificar a inversão monocular da profundidade, o julgamento escalar da profundidade ou relevo e a estimativa em centímetros da profundidade ou relevo da máscara côncava efetuadas por indivíduos com esquizofrenia. Tais objetivos se justificaram uma vez que não existem estudos do fenômeno da inversão visual da máscara côncava na condição de observação monocular envolvendo indivíduos com esquizofrenia. Outro objetivo específico colocado neste estudo foi verificar se o uso de medicação antipsicótica interferiria nesta inversão monocular da profundidade da máscara côncava. Justifica-se esse último objetivo em função de Schneider et al. (2002) observarem que a capacidade de inversão visual da profundidade dos indivíduos com esquizofrenia estaria restabelecida após três semanas de uso de antipsicótico.

\section{Método}

\section{Participantes}

Para o presente estudo, foram utilizadas três populações -alvo: duas clínicas e uma não-clínica. Todos os participantes demonstraram possuir acuidade visual de 6/6 na escala de Snellen e assinaram o Termo de Consentimento Livre e Esclarecido. A amostra da população-alvo clínica 1 foi constituída por sete indivíduos com esquizofrenia sob o uso de medicação antipsicótica por um período inferior ou igual a quatro semanas sendo três femininos e quatro masculinos, com média de idade de 33 anos $(D P=12,8)$. A amostra da população-alvo clínica 2 contou com 29 indivíduos com esquizofrenia sob uso de medicação antipsicótica por um período superior a quatro semanas sendo sete mulheres e 22 homens, com média de idade de 34 anos $(D P=8,1)$. A maioria das duas amostras sob o uso do antipsicótico típico Haloperidol. Dos 36 indivíduos das amostras da população-alvo clínica 1 e 2, 26 eram do sexo masculino (72\%) e 10 eram do sexo feminino (28\%) e possuíam média de idade de 34 anos $(D P=9,3)$, com período de escolarização menor ou igual a oito anos (75\%). Eles estavam sendo atendidos nos Serviços de Referência em Saúde Mental (SERSAM) na cidade de Divinópolis e Centro de Atenção 
Psicossocial (CAPS) Del Rey, em São João del Rei, M.G e preencheram os critérios para esquizofrenia segundo o Manual Diagnóstico e Estatístico de Transtornos Mentais, DSM-IV (2000) e Classificação Estatística Internacional de Doenças e Problemas Relacionados com a Saúde, CID-10 (1998).

Foram excluídos da amostra dos indivíduos da população -alvo clínicas 1 e 2 os indivíduos que apresentavam: comorbidade psiquiátrica grave, diagnóstico de retardo mental ou problema neurológico, impossibilidade de compreender as instruções do experimento, abstinência alcoólica, privação de sono por período prolongado, uso crônico de Cannabis Sativa e estado de crise psicótica no ato da realização do estudo.

A amostra da população-alvo não-clínica foi constituída por 29 indivíduos saudáveis sendo 21 do sexo feminino e oito do sexo masculino, com média de idade de 37 anos $(D P=11,8)$ que frequentavam o SERSAM e o CAPS Del Rey como acompanhantes dos pacientes.

\section{Material e equipamento}

Para a avaliação da percepção de profundidade monocular foi utilizada uma máscara de face confeccionada a partir de uma cabeça de uma boneca comercial de plástico, medindo $10 \times 6,5 \times 3 \mathrm{~cm}$ na qual foram demarcados os olhos, a boca e as sobrancelhas do lado côncavo ou oco, pintada com uma cor próxima à natural. A máscara côncava foi colocada no interior de uma caixa de $80 \mathrm{~cm}$ de comprimento, $37 \mathrm{~cm}$ de altura e $37 \mathrm{~cm}$ de largura, com um visor de $0,5 \mathrm{~mm}$ de diâmetro posicionado contralateralmente à mascara. Duas lâmpadas de $15 \mathrm{w}$ foram colocadas em cima e embaixo da máscara côncava dentro da caixa, conforme Figura 1. Utilizou-se a tabela de Snellen para verificar a acuidade visual dos participantes. Os olhos foram testados separadamente para, em seguida, serem avaliados em conjunto. Uma trena métrica com resolução em milímetros foi utilizada para estimar a medida de profundidade ou o relevo percebidos do reverso da máscara.

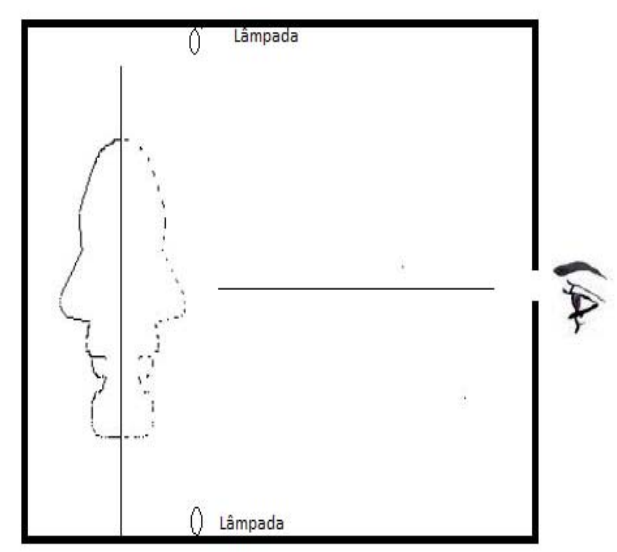

Figura 1: Desenho Esquemático da Caixa Utilizada na Pesquisa. A Linha Tracejada na Frente do Reverso da Máscara Representa a Percepção llusória da Face Possibilitada Pela Inversão da Profundidade Visual. Adaptado de Quaglia e Fukusima (2009).

\section{Procedimento}

Após a avaliação da acuidade visual, da assinatura do Termo de Consentimento Livre e Esclarecido e leitura das instruções, cada observador foi instruído a observar o reverso da máscara através do visor da caixa, com o olho preferencial.

A máscara côncava na posição vertical foi apresentada em ordem aleatória iluminada por cima ou iluminada por baixo. Em cada condição de apresentação da máscara côncava, foi solicitado ao participante relatar o relevo ou a profundidade percebida da máscara como côncava ou convexa e classificá-la de acordo com uma das seguintes categorias: (1) se percebida como muito côncava, (2) se percebida como côncava, (3) se percebida como plana, (4) se percebida como convexa e (5) se percebida como muito convexa. E por fim, solicitou-se que cada participante estimasse, em uma trena retrátil, a distância percebida entre a ponta do nariz e a base da máscara. $\mathrm{O}$ avaliador assinalava em uma folha as respostas dos participantes, isto é, as variáveis dependentes.

As informações concernentes aos objetivos e procedimentos do estudo foram asseguradas aos participantes da pesquisa. Foi apresentado e discutido o Termo de Consentimento Livre e Esclarecido. Foram dadas as informações sobre a duração do procedimento, o caráter confidencial das respostas e a possibilidade de interrupção da participação a qualquer momento. Também foi confirmado que as informações colhidas seriam sigilosas, que sua participação na pesquisa não interferiria no tratamento, e que a divulgação dos resultados seria anônima, aparecendo apenas os dados do grupo de participantes.

\section{Considerações éticas}

O presente estudo foi aprovado pela Comissão de Ética em Pesquisa Envolvendo Seres Humanos (CEPES), da Universidade Federal de São João del-Rei, conforme o memorando no 019/2012/UFSJ/CEPES e pelas coordenações do Serviço de Referência em Saúde Mental (SERSAM), em Divinópolis - MG, e do Centro de Atenção Psicossocial CAPS Del Rey, em São João del Rei - MG. As informações concernentes aos objetivos e procedimentos do estudo foram asseguradas aos participantes da pesquisa. Foi apresentado e discutido o Termo de Consentimento Livre e Esclarecido. Foram dadas as informações sobre a duração do procedimento, o caráter confidencial das respostas e a possibilidade de interrupção da participação a qualquer momento. Também foi confirmado que as informações colhidas seriam sigilosas, que sua participação na pesquisa não interferiria no tratamento, e que a divulgação dos resultados seria anônima, aparecendo apenas os dados do grupo de participantes.

\section{Resultados}

Os dados foram tratados estatisticamente pelo software Statiscal Package for the Social Sciences (SPSS) para Windows. $\mathrm{Na}$ análise estatística foi adotado o nível de significância $p<$ 
0,05 e o nível de $95 \%$ para o intervalo de confiança. Para a análise estatística dos dados foram utilizados os seguintes testes estatísticos: o teste de Kolmogorov-Smirnov para a verificação de normalidade dos dados e o teste de Levene para a verificação da homogeneidade das variâncias. Para a análise das variáveis dependentes, ainda foi utilizada a estatística U de Mann -Whitney e o teste $t$-Student para comparação das médias das estimativas da profundidade ou relevo da máscara côncava realizadas pelos indivíduos dos grupos em estudo. Também foram adotados o Teste Exato de Fisher para a verificação de independência das atribuições categóricas da máscara côncava efetuadas pelos indivíduos dos grupos em estudo e o teste Kruskal-Wallis, para a comparação das médias das estimativas efetuadas pelos indivíduos dos três grupos.

A análise dos dados revelou que as médias das idades estavam normalmente distribuídas, conforme o teste de Kolmogorov-Smirnov $(p>0,05)$ e eram homogêneas quanto à variância, conforme o teste de Levene $F(2,36)=0,13$; $(p>0,05)$. Adotando o teste $t$-Student verificou-se que não houve uma diferença estatisticamente significativa entre as médias das idades dos indivíduos saudáveis e indivíduos com esquizofrenia $t(63)=1,09 ;(p>0,05)$.

$\mathrm{Na}$ Tabela 1 são mostradas as frequências absolutas e porcentagens das atribuições categóricas da máscara côncava independente da direção da fonte de iluminação e do período de uso de medicação antipsicótica dos 29 indivíduos saudáveis e dos 36 indivíduos com esquizofrenia. $\mathrm{Na}$ análise estatística dos dados verificou-se que não houve diferença estatisticamente significativa $(p>0,05)$ entre as categorias atribuídas à máscara côncava efetuadas pelos indivíduos saudáveis e pelos indivíduos com esquizofrenia, pelo Teste Exato de Fisher.

Tabela 1

Frequências e Porcentagens das Atribuições Categóricas da Máscara Côncava Independente da Direção da Fonte de lluminação (Acima ou Abaixo) e do Período de Uso de Medicação Antipsicótica (Menos ou Mais de Quatro Semanas)

\begin{tabular}{lccccc}
\hline \multirow{2}{*}{ Indivíduos } & \multicolumn{4}{c}{ Categorias } & \\
\cline { 2 - 5 } & $\begin{array}{c}\text { Muito } \\
\text { Côncava }\end{array}$ & Côncava & Convexa & $\begin{array}{c}\text { Muito } \\
\text { Convexa }\end{array}$ & \\
\hline Saudáveis & 1 & 1 & 40 & 16 & 58 \\
& $(1,7 \%)$ & $(1,7 \%)$ & $(69,0 \%)$ & $(27,6 \%)$ & $(100 \%)$ \\
\hline \multirow{2}{*}{ Com Esquizofrenia } & 1 & 6 & 49 & 16 & 72 \\
& $(1,4 \%)$ & $(8,4 \%)$ & $(68,0 \%)$ & $(22,2 \%)$ & $(100 \%)$ \\
\hline
\end{tabular}

Na Tabela 2 são mostradas as frequências e porcentagens das atribuições categóricas à máscara côncava efetuadas pelos 29 indivíduos saudáveis, pelos sete indivíduos com esquizofrenia sob uso de medicação antipsicótica por um período inferior ou igual a quatro semanas e pelos 29 sob medicação antipsicótica por um período superior a quatro semanas. De maneira semelhante, verificou-se que não houve diferença estatisticamente significativa entre frequências das atribuições categóricas dos indivíduos saudáveis e dos indivíduos com esquizofrenia sob uso de medicação antipsicótica por período inferior ou igual a quatro semanas, tanto na condição de iluminação por cima da máscara $(p>0,05)$, quanto na condição de iluminação por baixo $(p>0,05)$, pelo Teste Exato de Fisher. De forma similar, na comparação entre os indivíduos saudáveis e os com medicação por período superior a quatro semanas, verificou-se, novamente pelo Teste Exato de Fisher, que não houve diferença estatisticamente significativa tanto na condição de iluminação por cima $(p>0,05)$ quanto na iluminação por baixo $(p>0,05)$.

\section{Tabela 2}

Frequências e Porcentagens das Categorias Percebidas Entre Indivíduos Saudáveis e Indivíduos com Esquizofrenia Sob Uso de Antipsicótico (Inferior ou Igual a Quatro semanas e Acima de Quatro semanas) nas Condições de lluminação por Cima e por baixo da Máscara Côncava

\begin{tabular}{|c|c|c|c|c|c|c|}
\hline \multirow{2}{*}{$\begin{array}{l}\text { Fonte de } \\
\text { Iluminação }\end{array}$} & \multirow[b]{2}{*}{ Indivíduos } & \multirow[b]{2}{*}{ 윯 잃 } & \multicolumn{2}{|c|}{ Categorias } & \multirow[b]{2}{*}{ 을 일 } & \multirow[b]{2}{*}{ Total } \\
\hline & & & 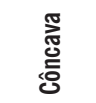 & ্ֻতু & & \\
\hline \multirow{3}{*}{ Por cima } & Saudáveis & $\begin{array}{c}1 \\
(3,5 \%)\end{array}$ & $\begin{array}{c}0 \\
(0,0 \%)\end{array}$ & $\begin{array}{c}19 \\
(65,5 \%)\end{array}$ & $\begin{array}{c}9 \\
(31,0 \%)\end{array}$ & $\begin{array}{c}29 \\
(100 \%)\end{array}$ \\
\hline & $\begin{array}{c}\text { Com } \\
\text { Esquizofrenia }\end{array}$ & $\begin{array}{c}0 \\
(0,0 \%)\end{array}$ & $\begin{array}{c}1 \\
(14,0 \%)\end{array}$ & $\begin{array}{c}6 \\
(85,7 \%)\end{array}$ & $\begin{array}{c}0 \\
(0,0 \%)\end{array}$ & $\begin{array}{c}7 \\
(100 \%)\end{array}$ \\
\hline & $\begin{array}{c}\text { Com } \\
\text { Esquizofrenia }\end{array}$ & $\begin{array}{c}0 \\
(0,0 \%)\end{array}$ & $\begin{array}{c}4 \\
(13,8 \%)\end{array}$ & $\begin{array}{c}20 \\
(69,0 \%)\end{array}$ & $\begin{array}{c}5 \\
(17,2 \%)\end{array}$ & $\begin{array}{c}29 \\
(100 \%)\end{array}$ \\
\hline \multirow{3}{*}{ Por baixo } & Saudáveis & $\begin{array}{c}0 \\
(0,0 \%)\end{array}$ & $\begin{array}{c}1 \\
(3,4 \%)\end{array}$ & $\begin{array}{c}21 \\
(72,4 \%)\end{array}$ & $\begin{array}{c}7 \\
(24,2 \%)\end{array}$ & $\begin{array}{c}29 \\
(100 \%)\end{array}$ \\
\hline & $\begin{array}{c}\text { Com } \\
\text { Esquizofrenia }\end{array}$ & $\begin{array}{c}1 \\
(14,3 \%)\end{array}$ & $\begin{array}{c}0 \\
(0,0 \%)\end{array}$ & $\begin{array}{c}5 \\
(71,4 \%)\end{array}$ & $\begin{array}{c}1 \\
(14,3 \%)\end{array}$ & $\begin{array}{c}7 \\
(100 \%)\end{array}$ \\
\hline & $\begin{array}{c}\text { Com } \\
\text { Esquizofrenia }\end{array}$ & $\begin{array}{c}1 \\
(3,4 \%)\end{array}$ & $\begin{array}{c}2 \\
(6,9 \%)\end{array}$ & $\begin{array}{c}20 \\
(69,0 \%)\end{array}$ & $\begin{array}{c}6 \\
(20,7 \%)\end{array}$ & $\begin{array}{c}29 \\
(100 \%)\end{array}$ \\
\hline
\end{tabular}

Na Figura 2 estão representadas as estimativas em centímetros da profundidade ou relevo da máscara côncava (independente da direção da fonte de iluminação) efetuadas pelos indivíduos saudáveis e com esquizofrenia (independente do período de uso de medicação antipsicótica). Na análise estatística verificou-se que houve diferença uma estatisticamente significativa $(p<0,05)$ entre as estimativas em cm da profundidade ou relevo da máscara côncava independente da direção da fonte de iluminação dos indivíduos saudáveis $(M=6,2 \mathrm{~cm}$, $E P=0,5)$ e dos indivíduos com esquizofrenia independentemente do período de uso de medicação antipsicótica $(M=5,4$ $\mathrm{cm}, E P=0,6$ ), pelo teste de Mann-Whitney. 


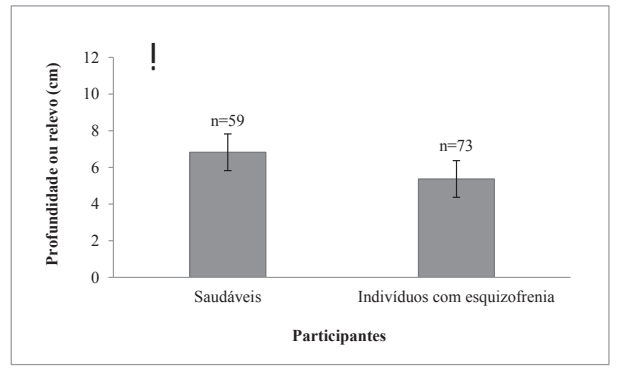

Figura 2: Estimativas Médias em cm da Profundidade ou Relevo Percebido da Máscara Côncava Independente da Direção Fonte de lluminação e do Período de Uso de Medicação Antipsicótica.

Na Figura 3 são mostradas as estimativas em centímetros da profundidade ou relevo da máscara côncava iluminada por cima dadas pelos indivíduos saudáveis, indivíduos com esquizofrenia sob uso de medicação antipsicótica por um período inferior ou igual a quatro semanas e pelos indivíduos com esquizofrenia sob uso de medicação antipsicótica por um pe- ríodo superior a quatro semanas. Na comparação das médias verificou-se não haver diferenças estatisticamente significativas entre as estimativas efetuadas pelos indivíduos saudáveis $(M=7,4 \mathrm{~cm}, E P=1)$ e indivíduos com esquizofrenia sob uso de medicação antipsicótica por um período inferior ou igual a quatro semanas $(M=7 \mathrm{~cm}, E P=1,3)$, no entanto, ao comparar os indivíduos saudáveis com o grupo de indivíduos com esquizofrenia sob uso de medicação antipsicótica por um período superior a quatro semanas (média $=5 \mathrm{~cm}, E P=1$ ), verificou-se uma diferença estatisticamente significativa $(p<0,05)$. Ainda nessa figura são mostradas as estimativas em centímetros da profundidade ou relevo da máscara côncava iluminada por baixo da máscara côncava efetuadas pelos indivíduos saudáveis $(M=6,2 \mathrm{~cm}, E P=0,8)$, indivíduos com esquizofrenia sob uso de medicação antipsicótica por um período inferior ou igual a quatro semanas $(M=8,3 \mathrm{~cm}, E P=1,8)$ e indivíduos com esquizofrenia sob uso de medicação antipsicótica por um período superior a quatro semanas $(M=4,6 \mathrm{~cm}, E P=0,7)$. Não houve, nessa condição de iluminação, diferença estatisticamente significativa pelo teste de Mann-Whitney.
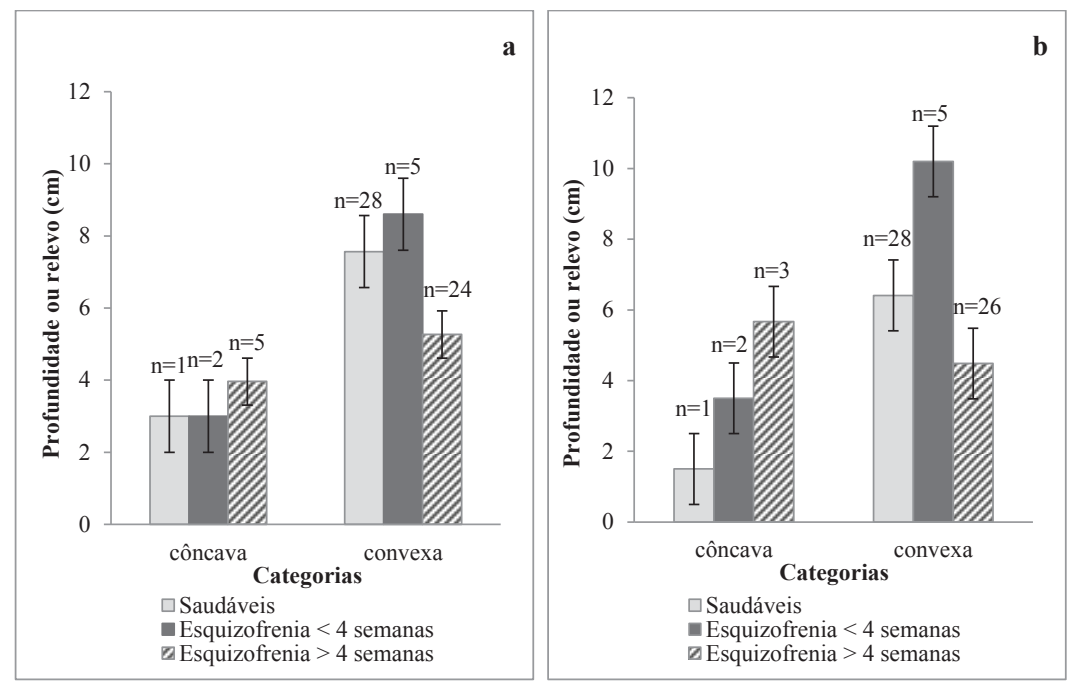

Figura 3: Estimativas Médias em cm da Profundidade ou Relevo da Máscara Côncava lluminada por Cima e por Baixo Efetuadas pelos Indivíduos Saudáveis, pelos Indivíduos com Esquizofrenia Sob Uso de Antipsicótico pelo Período Inferior ou Igual a Quatro Semanas e pelos Indivíduos com Esquizofrenia Sob Uso de Antipsicótico pelo Período Superior a 4 semanas. * $(p<0,05)$.

Os indivíduos saudáveis julgaram a profundidade ou relevo da máscara côncava em centímetros (média $=7,4 \mathrm{~cm}$; mediana $=6 \mathrm{~cm}$ ) e iluminada por baixo (média $=6,2 \mathrm{~cm}$; mediana $=5 \mathrm{~cm}$ ). Os indivíduos com esquizofrenia sob uso de antipsicótico por um período inferior ou igual a quatro semanas também julgaram em centímetros a profundidade ou relevo da com a máscara côncava iluminada por cima (média $=7 \mathrm{~cm}$; mediana $=7 \mathrm{~cm}$ ) e iluminada por baixo (média $=8,3 \mathrm{~cm}$; mediana $=7 \mathrm{~cm}$ ). A mesma atividade foi realizada pelos indivíduos com esquizofrenia sob uso de medicação antipsicótica por período superior a quatro semanas: a máscara côncava iluminada por cima (média $=5 \mathrm{~cm}$; mediana $=2,6 \mathrm{~cm}$ ) e iluminada por baixo (média $=4,6 \mathrm{~cm}$; mediana $=3,5 \mathrm{~cm}$ ). $\mathrm{Na}$ análise dos dados verificou-se uma diferença estatisticamente significativa ( $p$ $=0,03$ ) entre essas estimativas médias do relevo da máscara côncava dos três grupos quando iluminada por cima através do teste Kruskal-Wallis.

\section{Discussão}

A maioria dos indivíduos com esquizofrenia realizou a inversão da profundidade da máscara côncava na condição de observação monocular. A direção da fonte de iluminação não foi um fator determinante para a inversão visual da profundidade. Este resultado está em consonância com os resultados obtidos 
no estudo de Quaglia e Fukusima (2009) onde, embora a pesquisa tenha sido conduzida com estudantes universitários, as variações na direção da fonte de iluminação da máscara côncava não interferiram na inversão monocular da profundidade. Esses autores utilizaram uma máscara de tamanho natural inserida no interior de uma câmara, observada monocularmente através de um visor. Na presente pesquisa foram recriadas condições semelhantes, porém com dimensões reduzidas. Utilizou-se uma máscara côncava confeccionada de uma face de boneca de plástico que foi introduzida no interior de uma caixa e também observada monocularmente através de um visor. A máscara côncava foi iluminada aleatoriamente por cima ou por baixo da cabeça. A máscara côncava utilizada nesta pesquisa foi apresentada na condição de observação monocular iluminada ora por cima, ora por baixo e em tal condição os indivíduos com esquizofrenia perceberam-na como convexa. Este resultado é diverso dos resultados obtidos nos estudos de Schneider et al. (1996; 2002), por Koethe et al. (2009) e por Dima et al. (2009; 2010; 2011), nos quais os indivíduos com esquizofrenia sofreram prejuízos na realização da inversão binocular da profundidade. $\mathrm{Na}$ atual pesquisa, a maioria dos indivíduos com esquizofrenia atribuiu a categoria convexa à mascara côncava. As condições de apresentação dos estímulos, monocular e binocular, e a natureza dos estímulos, uma máscara tridimensional na presente pesquisa e imagens bidimensionais apresentadas estereoscopicamente nos demais estudos, podem ter contribuído para essas diferenças nos resultados. O estímulo tridimensional gera, por si só, indícios de profundidade, diferente das imagens bidimensionais. A máscara côncava, de tamanho natural objetiva apresentada na condição de observação monocular, requer distâncias menores entre a máscara e o observador para a inversão visual da profundidade do que a sua apresentação na condição binocular (Hill \& Bruce, 1993). Com a apresentação monocular, perdem-se as informações decorrentes da disparidade binocular ou retiniana, importantes para se avaliar a profundidade do estímulo (Sternberg, 2008). Na presente pesquisa, no entanto, não foram requeridas distâncias para a inversão monocular da profundidade da máscara côncava. Os participantes observaram o estímulo através de um visor a partir de um ponto de vista estacionário.

A máscara côncava utilizada nesta pesquisa continha propriedades e atributos, como as demarcações em cores da boca, olhos, sobrancelhas, que seriam integradas pelo sistema visual e organizadas por mecanismos de síntese cerebrais (Lent, 2005) resultando na percepção de sua forma côncava. Esses atributos utilizados não foram suficientes para que os indivíduos a percebessem como côncava, conforme sua configuração real. De fato, Hill e Johnston (2007) já tinham alertado para o fato da coloração do reverso da máscara com tonalidades próximas às da face humana facilitar a inversão binocular da profundidade de indivíduos saudáveis. Provavelmente, um processo de geração de hipóteses sobre a forma tridimensional de faces mediado pelos conhecimentos conceitual e perceptivo, presentes no processamento de cima para baixo, top-down, na presente pesquisa, se sobrepôs aos estímulos sensoriais que chegaram ao cérebro, processo bottom-up (Gregory, 2007).

O período de uso de medicação antipsicótica foi outra variável não determinante para a inversão monocular da profundidade da máscara côncava por indivíduos com esquizofrenia. Não foram observadas diferenças estatisticamente significativas para a inversão monocular da profundidade da máscara côncava iluminada por cima ou por baixo entre os indivíduos com esquizofrenia sob o uso de medicação antipsicótica por período inferior ou igual e superior a quatro semanas. Diversamente, e com observação binocular, os estudos a seguir não levaram em consideração a direção da fonte de iluminação sobre o estímulo, mas avaliaram o período de utilização da medicação antipsicótica sobre a inversão visual da profundidade. Koethe et al. (2009) observaram que indivíduos com esquizofrenia sem tratamento medicamentoso e indivíduos com esquizofrenia com nove dias de período de tratamento com antipsicótico tiveram prejuízo na realização da inversão binocular da profundidade de imagens estereoscópicas de faces de homens de meia-idade. Resultados semelhantes foram obtidos por Schneider et al. (2002) com indivíduos com esquizofrenia sob tratamento com antipsicótico durante a primeira e terceira semanas. Os indivíduos saudáveis que participaram da pesquisa realizaram a inversão binocular da profundidade de imagens estereoscópicas da face humana, mas o mesmo não ocorreu com os indivíduos com esquizofrenia, com uma e três semanas de uso de medicação antipsicótica. No momento da alta hospitalar, os indivíduos com esquizofrenia foram capazes de realizar a inversão binocular da profundidade da máscara. Uma possível explicação para as diferenças entre esses resultados dizem respeito às condições de apresentação do estímulo, binoculares nas pesquisas de Koethe et al. (2009) e Schneider et al. (2002) e monocular na presente pesquisa. É possível que a condição de observação monocular também favoreça a inversão visual da profundidade da máscara côncava em indivíduos com esquizofrenia, semelhante ao observado com indivíduos saudáveis por Hill e Bruce (1993). Por outro lado, os indivíduos com esquizofrenia do grupo inferior ou igual a quatro semanas desta pesquisa já haviam iniciado o uso de medicação antipsicótica. Pode ter ocorrido uma sinergia entre a condição de observação monocular e o uso de medicação antipsicótica favorecendo a inversão da profundidade da máscara côncava. Ademais, não foi utilizada uma escala para medir a severidade dos sintomas da esquizofrenia.

A análise dos dados quantitativos revelou uma diferença estatisticamente significativa entre as estimativas médias em $\mathrm{cm}$ da profundidade ou relevo da máscara côncava efetuada pelos indivíduos saudáveis e pelos indivíduos com esquizofrenia independente da direção da fonte de iluminação e do período de uso de antipsicótico. Constatou-se, também, uma diferença estatisticamente significativa entre as médias das estimativas da máscara côncava para os três grupos, quando a mesma foi iluminada por cima. Indivíduos com esquizofrenia sob uso de antipsicótico por um período superior a quatro semanas estimaram o relevo da máscara côncava em menor comprimento comparados aos indivíduos saudáveis e aos 
indivíduos com esquizofrenia sob uso de antipsicótico por um período inferior ou igual a quatro semanas. É possível que o de uso de antipsicótico por um período superior a quatro semanas provoque essa compressão na percepção da profundidade ou relevo da máscara côncava observada monocularmente. Noll (2007) elencou como uma das características da esquizofrenia a perda da percepção da profundidade visual, que faz com que o mundo pareça achatado, bidimensional. Mas não há, na literatura científica, estudos que comparem as estimativas da profundidade ou relevo percebido da máscara côncava efetuadas por indivíduos saudáveis e efetuadas por indivíduos com esquizofrenia sob diferentes períodos de uso de antipsicótico na condição de observação monocular.

Esta pesquisa apresenta a limitação de generalização dos resultados para população de indivíduos com esquizofrenia. Os participantes não foram selecionados aleatoriamente da população-alvo e, desse modo, reduz-se a possibilidade de sua generalização (Selltiz, Wrightsman, \& Cook, 1987). Outra limitação deste estudo refere-se à diferença entre os tamanhos amostrais dos grupos clínicos. A investigação foi realizada na condição de observação monocular e foi utilizada como estímulo uma máscara côncava confeccionada de uma face de boneca de tamanho reduzido. Sugerem-se novas investigações sobre a percepção da máscara côncava de tamanho reduzido iluminada em diferentes direções, com observação monocular e binocular, em indivíduos saudáveis e com esquizofrenia sob diferentes períodos de medicação antipsicótica. Sugere-se, ainda, investigar uma possível relação entre o grau de severidade da esquizofrenia com a inversão monocular da profundidade da máscara côncava.

\section{Conclusões}

Os indivíduos com esquizofrenia sob uso de medicação antipsicótica pelos períodos inferior ou igual e superior a quatro semanas realizaram a inversão monocular da profundidade da máscara côncava. O período de uso de medicação antipsicótica não foi relevante na realização da inversão monocular da profundidade da máscara côncava. No entanto, os indivíduos com esquizofrenia sob uso de medicação antipsicótica pelo período superior a quatro semanas estimaram o relevo da máscara côncava iluminada por cima em menor comprimento, comparados aos indivíduos saudáveis e aos indivíduos com esquizofrenia sob uso de medicação antipsicótica pelo período inferior ou igual a quatro semanas. O uso de medicação antipsicótica por um período mais prolongado parece comprimir a profundidade percebida da máscara côncava em indivíduos com esquizofrenia.

\section{Referências}

Associação Americana de Psiquiatria. (2000). Manual Diagnóstico e Estatístico de Transtornos Mentais - DSM-IV (4⿳亠丷a ed. rev.). Porto Alegre: Artmed.

Cruz, E. D. N. (2010). Efeitos do alcoolismo na percepção visual de contraste (Dissertação de mestrado). Universidade Federal de Pernambuco, Recife, PE, Brasil. Recuperado de http://www.ufpe.br/pospsicologia/images/ Dissertacoes/2010/cruz\%20llen\%20dias\%20niccio\%20da.pdf
Dima, D., Dietrich, D. E., Dillo, W. \& Emrich, H. M. (2010). Impaired top-down process in schizophrenia: A DCM study of ERPs. Neuroimage, 52, 824-832. doi:10.1016/j.neuroimage.2009.12.086

Dima, D., Dillo, W., Bonnemann, C., Emrich, H. M., \& Dietrich, D. E. (2011) Reduced P300 and P600 amplitude in the hollow-mask illusion in patients with schizophrenia. Psychiatry Research: Neuroimaging, 191(2), 145-151. doi: 10.1016/j.pscychresns.2010.09.015

Dima, D., Roiser, J. P., Dietrich, D. E., Bonnemann, C., Lanfermann, H., Emrich, H. M... Dillo, W. (2009). Understanding why patients with schizophrenia do not perceive the hollow-mask illusion using dynamic causal modeling. Neuroimage, 46, 1180-1186.

Gregory, R. L. (1997). Knowledge in perception and illusion. Philosophical Transactions of the Royal Society London Series B: Biological Sciences, 352, 1121-1128.

Hill, H., \& Bruce, V. (1993). Independent effects of lighting, orientation, and stereopsis on the hollow-face illusion. Perception, 22, 887-897. doi:10.1068/p220887

Hill, H., \& Johnston, A. (2007). The hollow-face illusion: Object-specific knowledge, general assumptions or properties of stimulus? Perception, $36(2), 199-223$

Kleffner, D., \& Ramachandran, V. S. (1992). On the perception of shape from shading. Perception \& Psychophysics, 52, 18-36. Recuperado de http://cbc. ucsd.edu/pdf/Shape_From_Shading_P_\&_P.pdf

Koethe, D., Kranaster, L., Hoyer, C., Gross, S. Neatby, M. A., Schultze-Lutter, ... Leweke, F. M. (2009). Binocular depth inversion as a paradigm of reduced visual information processing in prodromal state, antipsychotic-naïve and treated schizophrenia. European Archives of Psychiatry and Clinical Neuroscience, 259, 195-2002. doi: 10.1007/s00406-008-0851-6

Lent, R. (2005). Cem bilhões de neurônios: Conceitos fundamentais de neurociências. São Paulo: Editora Atheneu.

Noll, Richard (2007). The Encyclopedia of Schizophrenia and Other Psychotic Disorders. (3ㄹed.). Nova Iorque: Facts On File.

Organização Mundial de Saúde. (1998). CID-10: Classificação Internacional de Doenças e Problemas Relacionados à Saúde (10ª ed.). São Paulo: Edusp.

Quaglia, M. A. C., \& Fukusima, S. S. (2009). Cor, iluminação e orientação do reverso de uma máscara facial não afetam a ilusão da máscara côncava. Estudos de Psicologia,14(2), 97-105. 10.1590/S1413-294X2009000200002

Schneider, U., Borsurtzky, M., Seifert, J., Leweke, F. M., Huber, T. J., Rollnik, J. D., \& Emrich, H. M. (2002). Reduced binocular depth inversion in schizophrenic patients. Schizophrenia Research, 53, 101-108. doi:10.1016/ S0920-9964(00)00172-9

Schneider, U., Leweke, F. M., Sternemann, Weber, M. M., \& Emrich, H. M. (1996). Visual 3D illusion: A systems-theoretical approach to psychosis. European Archives of Psychiatry and Clinical Neuroscience, 246(5), 256-260. doi:10.1007/BF02190277

Selltiz., C., Wrightsman, L. S., \& Cook, S. W. (1987). Métodos de pesquisa nas relações sociais: Delineamentos de pesquisa (Vol. 1). São Paulo: Editora da USP.

Sternberg, R. J. (2008). Psicologia cognitiva. (4ª ed.). Porto Alegre: Artmed.

Walther, S., Federspel, A., Horn, H., Bianchi, P., Wiest, R., Wirth, M., ... Müller J. T. (2009). Encoding deficit during face processing within the right fusiform face area in schizophrenia. Psychiatry Research, 17(3), 184-191. doi: 10.1016/j.pscychresns.2008.07.009

Yoshida, H. (2006). The effects of facial texture, stimulus orientation and light direction on the hollow-face illusion. Bulletin of the Graduate School of Education, Hiroshima University, Education and Human Science, 55, 321329. 
Arthur Alves, Mestre em Psicologia pela Universidade Federal de São João Del-Rei - UFSJ, é Psicólogo na escola municipal "Raio de Sol” em Divinópolis-MG. Endereço para correspondência: Rua Campos Sales, 465, Bairro Porto Velho- Divinópolis-MG. Telefone: (37)3222-1796. E-mail: arthuralvespsicologo@bol. com.br

Maria Amélia Cesari Quaglia, Doutora em Psicobiologia pela Faculdade de Filosofia, Ciências e Letras de Ribeirão Preto (FFCLRP - USP), é Professora Associada II da Universidade Federal de São João del Rei (UFSJ). E-mail: melinha@ufsj.edu.br

Livia da Silva Bachetti, Mestre em Psicologia pela Universidade Federal de São João del Rei (UFSJ), é doutoranda em Psicobiologia na Universidade de São Paulo (FFCLRP - USP) e Psicóloga. E-mail: livbachetti@yahoo.com.br

Marcos Santos de Oliveira, Doutor em Estatística e Experimentação Agropecuária pela Universidade Federal de Lavras (UFLA), é Professor Adjunto II na Universidade Federal de São João del Rei (UFSJ). E-mail: mso@ufsj.edu.br 